\title{
How can the Nanostructure Affect the Charge Transport in
}

\author{
PLED? \\ M M D Ramos ${ }^{\mathrm{a}}$ and H M C Barbosa \\ Department of Physics, University of Minho, \\ Campus de Gualtar, 4710-057 Braga, Portugal \\ Tel.: +351 253604 330; fax: +351 253678981 \\ E-mail address: marta@ fisica.uminho.pt (M. M. D. Ramos) \\ helder@fisica.uminho.pt (H. M. C. Barbosa)
}

\begin{abstract}
In polymer light emitting diodes (PLEDs) each semiconducting polymer chain consists of a large number of conjugated segments linked by kinks or twists and each one of them behaves like a separated straight strand. The length and orientation of the conjugated strands relative to the electrodes surface depend on the deposition conditions used. Atomistic results have shown that the molecular properties of the conjugated strands depend on their length, which can affect the electronic processes involved in PLEDs. The aim of this work is to study the influence of the average conjugation length within the polymer layer on charge injection, trapping and recombination in PLEDs for all polymer strand orientations relative to the electrodes surface obtained experimentally by different techniques. For that purpose we use a mesoscopic model that considers the morphology and the molecular properties of the polymer. Our results show that by increasing the average conjugation length of the active polymer layer the amount of charge injected into
\end{abstract}


the device increases and the recombination probability occurs preferentially in segments longer than the average conjugation length, both effects having implications on the performance of polymer LEDs.

${ }^{a}$ Corresponding author

\section{PACS: 73.23.-b, 72.20. Jv, 73.40. Sx}

\section{Introduction}

One of the advantages of using conjugated polymers in light emitting diodes, when compared to low weight organic molecules, is the possibility of processing them in solution, which allows the deposition of these polymers onto large-area substrates at room temperature and pressure, using different wet-processing techniques. The solid state polymer film presents a nanostructure where the conjugated polymer chains have kinks and twists that break their conjugation creating several straight conjugated segments, which can act as individual optical chromophores [1]. The interaction between the conjugated segments depends on the solvent used and the film-processing conditions. A polymer chain can be folded on itself creating an ensemble of stiff-straight segments that presents a height degree of alignment or have an unfolded structure where the straight conjugated segments are randomly oriented. There are clear evidences that orientation of the conjugated segments relative to the electrodes surface within the highly-oriented domains and/or the size of the amorphous phase depend how the polymer deposition is made or the treatment of the polymer film thereafter. Ink-jet printing of poly(p-phenylene 
vinylene) (PPV) derivatives leads to a nanostructure where the stiff-chain segments are preferentially oriented perpendicular to the substrate [1], while spin-coating deposition leads to an orientation of the segments axis parallel to the subtrate [2]. The increase of the amorphous phase can be achieved by thermal annealing of the polymer layer. This last strategy, which is used to improve the long-term stability of the device, leads to an open structure of the polymer chains where the stiff-chain segments have a random orientation [3]. Independently of the deposition technique or the treatment used, the average conjugation length of the segments within the polymer layer can vary and this will have implication in the device efficiency since the molecular properties of the conjugated strands depend on their length [4]. In this paper we present a theoretical study of the effect of the average conjugation length on charge injection, trapping and recombination in a polymer light emitting diode (PLED) with a PPV layer as the active component. We have considered here the three different polymer morphologies that can be obtained by the experimental techniques mentioned above.

\section{Model and computer simulation details}

To model the functioning of a PLED, we consider a single polymer layer with 100 nm thickness sandwiched between two planar electrodes, and both electrodes present no impurities or defects at their surface.

The polymer layer consists of PPV straight rigid conjugated segments with a Gaussian distribution of lengths and a mean value varying from 5 to 7 monomers. For each one of the average conjugation length considerer in this work, we built polymer films with three different types of morphology corresponding to straight rigid segments 
parallel, perpendicular and randomly oriented relative to the electrodes surface. A minimum intermolecular distance of $0.650 \mathrm{~nm}$ was considered, based on self-consistent quantum molecular dynamics calculations [5], and the maximum film density obtained for the random morphology $(0.3 \mathrm{~g} / \mathrm{cm} 3)$ was also used for parallel and perpendicular morphologies.

Our model of bipolar charge transport in PLED is based on a generalized Monte Carlo algorithm which takes into account the competition between the processes of charge injection, transport, trapping, recombination and collection by the opposite electrodes. To simulate bipolar charge injection, we consider that the injection process occurs by hopping. The charge (electron or hole) injection probability takes into account the distance between the electrode and the polymer strand, as well as the potential barrier. In the absence of an applied electric field, the potential barrier height (zero-field barrier height) is equal to the difference between cathode/anode work function and the electron affinity (EA)/ionization potential (IP) (i.e. the molecular properties) of the polymer strand obtained from atomistic calculations [4]. When an electric field is applied the potential barrier height changes due to the local electric field (i.e the sum the applied electric field, the field due to space charge distribution through the polymer network and the field that results from the electrodes polarization). A detailed description of the injection process can be found in [6].

Our nanoscale model of charge transport, which is described in detail elsewhere [7], considers two processes both depending on the local electric field. The first process, known as intramolecular mobility, occurs when a charge is injected into a polymer strand and its electric field dependence was obtained by self-consistent quantum molecular 
dynamics calculations performed by Ramos and co-workers [8]. The interaction between the charge and polymer strand leads to a formation of a quasi-particle known as polaron that will move towards the centre of the conjugated segment if the local electric field along the segment axis is smaller than a certain threshold obtained from atomistic calculations [8], otherwise it will move towards one of the segment ends, depending on the charge sign and the direction of the local field. The second process, known as intermolecular mobility occurs when a charge jumps between different polymers strands by a thermally activated process known as hopping. The calculated probability for an electron/hole to hop between two neighbouring strands depends on the local electric field, the molecular properties of the polymer strands and the distance between hopping sites. When a charge percolates along the polymer network it can be stored or trapped in a conjugated segment, remaining there until the local electric field allows it to hop to a neighbour segment. During the hopping process, an injected charge can reach the electrode opposite to the injection one, being collected by it and contributing to the electric current, or it can meet a charge of opposite sign in a polymer strand, and recombine if the local electric field along the molecular axis is not strong enough to keep both charges apart.

\section{Results and discussion}

To study the influence of the average conjugation length in the functioning of PLEDs, we performed computer experiments using PPV layers with the axis of the polymer strands parallel, perpendicular or randomly oriented relative to the electrodes surface. All simulations were performed for external applied electric fields lower (1 
$\mathrm{MV} / \mathrm{cm})$ and higher $(3 \mathrm{MV} / \mathrm{cm})$ than the threshold for intramolecular mobility of electrons (1.55 MV/cm) and holes (2.0 MV/cm) [8], and ohmic electric contacts at both electrode/polymer interfaces which were obtained by adjusting the Fermi level of the electrodes to bring them close to the average ionization potential/electron affinity of the conjugated segments near the anode/cathode. Consequently charge injection rate is only limited by space charge effects.

Fig. 1 shows the total amount of injected charges in layers with different polymer morphology as a function of the average conjugation length, at the end of a certain number of iterations and after the steady state is reached. The amount of charges injected is higher for the applied electric field is $3 \mathrm{MV} / \mathrm{cm}$ regardless of the average conjugation length, being almost zero for the perpendicular morphology when the applied electric field is of $1 \mathrm{MV} / \mathrm{cm}$. These results are consequence of different contribution of the local electric field for the charge hopping process between two strands and for the intramolecular charge mobility. For the perpendicular morphology the hopping sites are parallel to the electrodes surface and perpendicular to the applied electric field. As a result when the applied electric field is smaller than the field needed for intramolecular charge mobility most of the injected charges will stay trapped in the middle of the strands near the electrode, hindering the injection of new charges. With the increase of the applied electric field to $3 \mathrm{MV} / \mathrm{cm}$, charges can easily move along strands, since now the local electric field along the molecular axis is higher than the threshold for intramolecular mobility. When the charge reaches the strand end favoured by the local electric field, it can hop to other strand in the direction perpendicular to the electrodes surface because the field strength is strong enough to decrease the barrier height for charge hopping. In 
the case of the parallel morphology the preferential hopping direction between two neighbouring strands is parallel to the applied electric field, which allows a maximum contribution of this field to the hopping process. For this morphology a charge can only have intramolecular mobility due to the electric field created by the distribution of the other injected charges within the polymer layer, but this effect is negligible for the transport of the injected charge towards the opposite electrode. The random orientation of the conjugated segments within the polymer network allows a contribution of the applied electric field to the hopping process as well as the intramolecular mobility, both processes contributing to move the charges away from the injection electrode. The local electric field needed for intramolecular mobility has also the contribution of the field created by the distribution of the injected charges.

As a result the amount of charges injected in the random morphology is higher than for parallel and perpendicular morphologies. Since intramolecular mobility is a much faster process than intermolecular mobility, the increase of the applied electric field above the threshold needed for a charge to move along the conjugated strands will have a higher impact in charge transport, and consequently in charge injection, at high applied bias for the random and perpendicular morphologies than for the parallel morphology. Therefore, the increase of the applied electric field leads to an increase in the total amount of injected charge for all polymer morphologies, the effect being more pronounced for random and perpendicular morphologies.

When the average conjugation length within the polymer layer increases from 5 to 7 monomers, the contribution of the intramolecular mobility to charge transport towards the opposite electrode at high applied electric field only increases for the random and 
perpendicular morphologies. As a result the amount of injected charges for the parallel morphology does not seem to change with the conjugation length whereas it increases with the increase of the conjugation length for the random and perpendicular morphologies. This effect is more pronounced for the perpendicular morphology.

Although there is an increase in the amount of injected charge with the increase of the strength of the applied electric field, our results show that the storage of the charge (electrons and holes) per strand length within the polymer layer at steady state does not depend on the polymer morphology (see Fig. 2 for the hole distribution which is similar for the electron distribution).

For an average conjugation length of 5 monomers holes are stored or trapped preferentially in segments with 6 monomers and there are more holes stored in segments with longer lengths than with shorter ones, regardless of the polymer morphology. We can then conclude that holes and electrons distribution per strand length within the polymer layer depends on the molecular properties of the polymer segments and not the way that they are oriented within the layer. Since the ionization potential decreases and the electron affinity increases with the increase of the conjugation length, holes and electrons will hop more easily to longer segments than to the shorter ones.

Therefore, a similar result was obtained for both electrons and holes at low applied electric fields (e.g. $1 \mathrm{MV} / \mathrm{cm}$ ). By increasing the average conjugation length within the polymer layer, the profiles for hole distribution per segment length remains the same but they are shifted towards longer conjugation lengths (see Fig. 3). The Gaussiantype profile obtained for electron and hole distribution per segment length seems to be related to the Gaussian distribution of stiff-chain lengths used in our nanoscale model. 
The change in the average conjugation length of the polymer layer also affects the distribution of charge recombination events per strand length. Fig. 4 shows that the distribution of the recombination events per segment with length is similar to those for charge storage at the steady state for different average conjugation lengths and the same polymer morphology. As in the case of charge distribution, the fraction of recombination events is also higher in segments with lengths longer than the average conjugation length than in the shorter ones.

In contrast to charge distribution, the maximum number of recombinations takes place at strands with the same length as the average conjugation length of the polymer layer. The behaviour described above for the distribution of recombination events per strand length does not depend on the polymer morphology and the strength of the applied electric field.

Since the increase of charge injection leads to an increase of the number of recombination events and the probability of light emission seems to increase with the increase of conjugation length [9], the results of this work suggest that device performance is sensitive to both the morphology of the polymer film and its average conjugation length, which can be controlled by changing solvents and fabrication conditions $[10,11]$.

\section{Conclusions}

Understanding how the morphology and the average conjugation length of the polymer layer affects PLED performance is one of the key issues for the advancing of the nanoelectronics technology. Using a nanoscale modelling we have demonstrated that 
changes in the length of the polymer molecules and their molecular arrangements can affect a polymer LED functioning. Our results show that the effect of the nanostructure in charge injection and consequently in charge recombination, is more pronounced for an applied electric field higher than the threshold for intramolecular mobility and for long average conjugation length. The distribution of charge and recombination events per segment length do not depend on the molecular arrangement within the polymer layer but depends on its average conjugation length which affects the molecular properties. Although our nanoscale model is a simple approximation to the complex structure of a semiconducting polymer bulk, these results can shed some light on the influence of the nanostructure in a polymer LED and they suggest that molecular arrangements relative to the electrodes and the average conjugation length are very important factors in controlling device performance.

\section{Acknowledgement}

This work is part of the research projects POCTI/CTM/41574/2001 and CONCREEQ/443/EEI/2005, approved by the Portuguese Foundation for Science and Technology (FCT) and support by the European Community Fund FEDER. One of us (H.M.C.B.) is also indebted to $\mathrm{FCT}$ for financial support under $\mathrm{PhD}$ grant $\mathrm{N}^{\circ}$ SFRH/BD/22143/2005. 


\section{References}

[1] B. G. Sumpter, P. Kumar, A. Mehta, M. D. Barnes, W. A. Shelton, and R. J. Harrison, J. Phys. Chem. B 109. 7671 (2005)

[2] C. Y. Yang, F. Hide, M. A. Diaz-Garcia, A. J. Heeger, and Y. Cao, Polymer 39. $2299(1998)$

[3] J. Kim, J. Lee, C. W. Han, N. Y. Lee, and I. J. Chung, Appl. Phys. Lett. 82. 4238 (2003)

[4] A. M. Stoneham, M. M. D. Ramos, A. M. Almeida, H. M. G. Correia, R. M. Ribeiro, H. Ness, and A. J. Fisher, J. Phys.-Condes. Matter 14. 9877 (2002)

[5] M. M. D. Ramos, A. M. Almeida, H. M. G. Correia, R. M. Ribeiro, and A. M. Stoneham, 238. 438 (2004)

[6] H. M. C. Barbosa and M. M. D. Ramos, Plasma Process. Polym. 4. S104 (2007)

[7] M. M. D. Ramos and H. M. G. Correia, J. Phys.: Condens. Matter 18. S429 (2006)

[8] A. M. Almeida, M. M. D. Ramos, and H. G. Correia, Comput. Mater. Sci. 27. 128 (2003)

[9] H. M. G. Correia, PhD Thesis, University of Minho, 2007

[10] J. Liu, Y. J. Shi, L. P. Ma, and Y. Yang, J. Appl. Phys. 88. 605 (2000)

[11] T. Q. Nguyen, V. Doan, and B. J. Schwartz, J. Chem. Phys. 110. 4068 (1999) 


\section{List of Figures Captions:}

Figure 1 - Total number of charges (electrons and holes) injected into the PPV layers with random (squares), parallel (triangles) and perpendicular (circles) morphologies, for an applied electric field of $1 \mathrm{MV} / \mathrm{cm}$ (closed marks) and $3 \mathrm{MV} / \mathrm{cm}$ (open marks). The lines are just a guide to the eyes.

Figure 2 - Fraction of the total number of holes stored in polymer segments as a function of their length, in PPV layers with random (squares), parallel (triangles) and perpendicular (circles) morphologies. These results were obtained for an applied electric field of $3 \mathrm{MV} / \mathrm{cm}$ and an average conjugation length of 5 monomers. The lines are just a guide to the eyes.

Figure 3 - Fraction of the total number of holes stored in polymer segments of different lengths when the average conjugation length in the polymer layer is of 5 (squares), 6 (circles) and 7 (triangles) monomers. These results were obtained for a PPV layer with random morphology and an applied electric field of $3 \mathrm{MV} / \mathrm{cm}$. The lines are just a guide to the eyes.

Figure 4 - Fraction of the total number of charges that undergoes recombination in polymer segments with different lengths when the average conjugation length in the polymer layer is of 5 (squares), 6 (circles) and 7 (triangles) monomers. These results were obtained for a PPV layer with random morphology and an applied electric field of 3 $\mathrm{MV} / \mathrm{cm}$. The lines are just a guide to the eyes. 
Figure 1

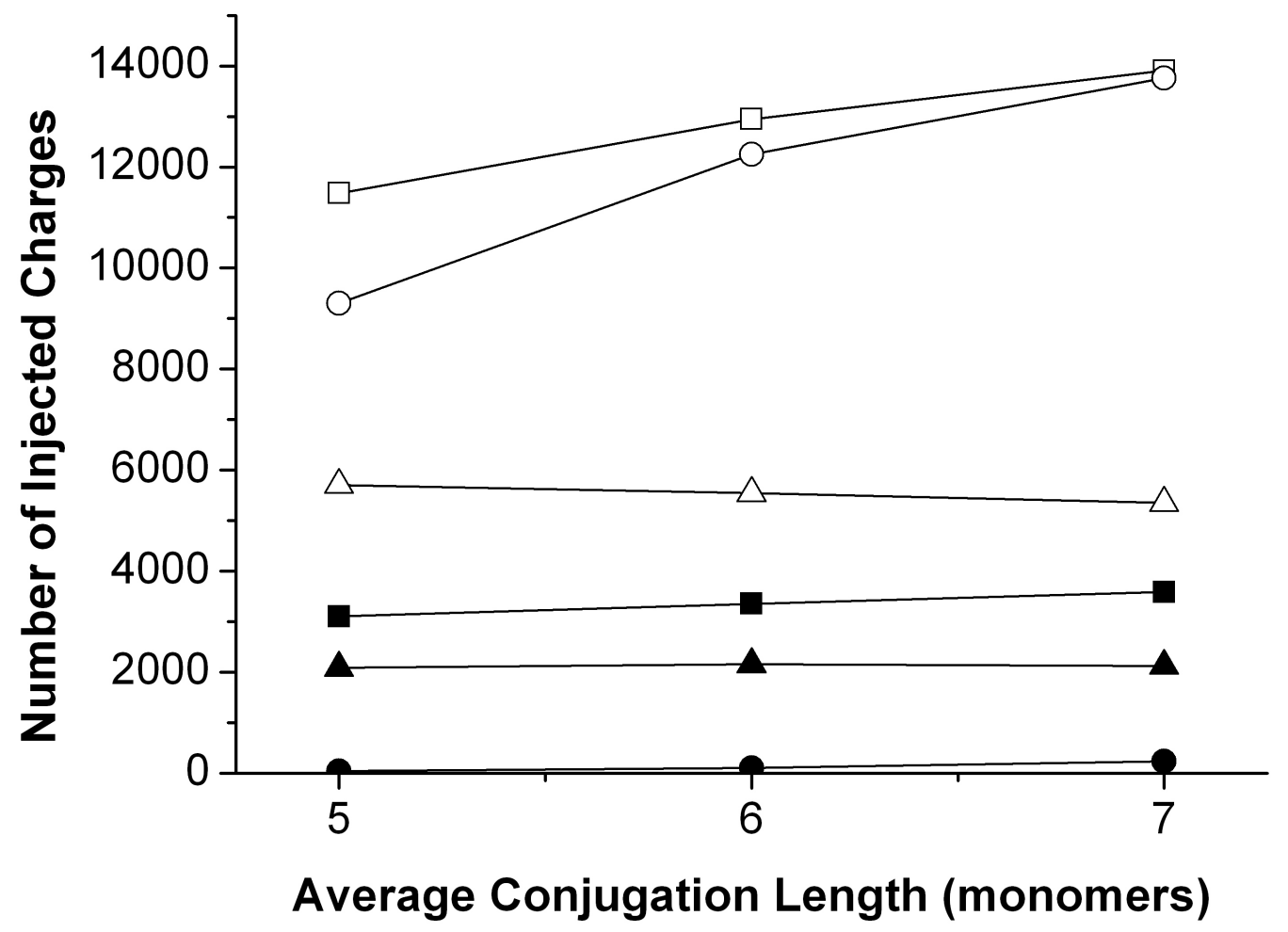


Figure 2

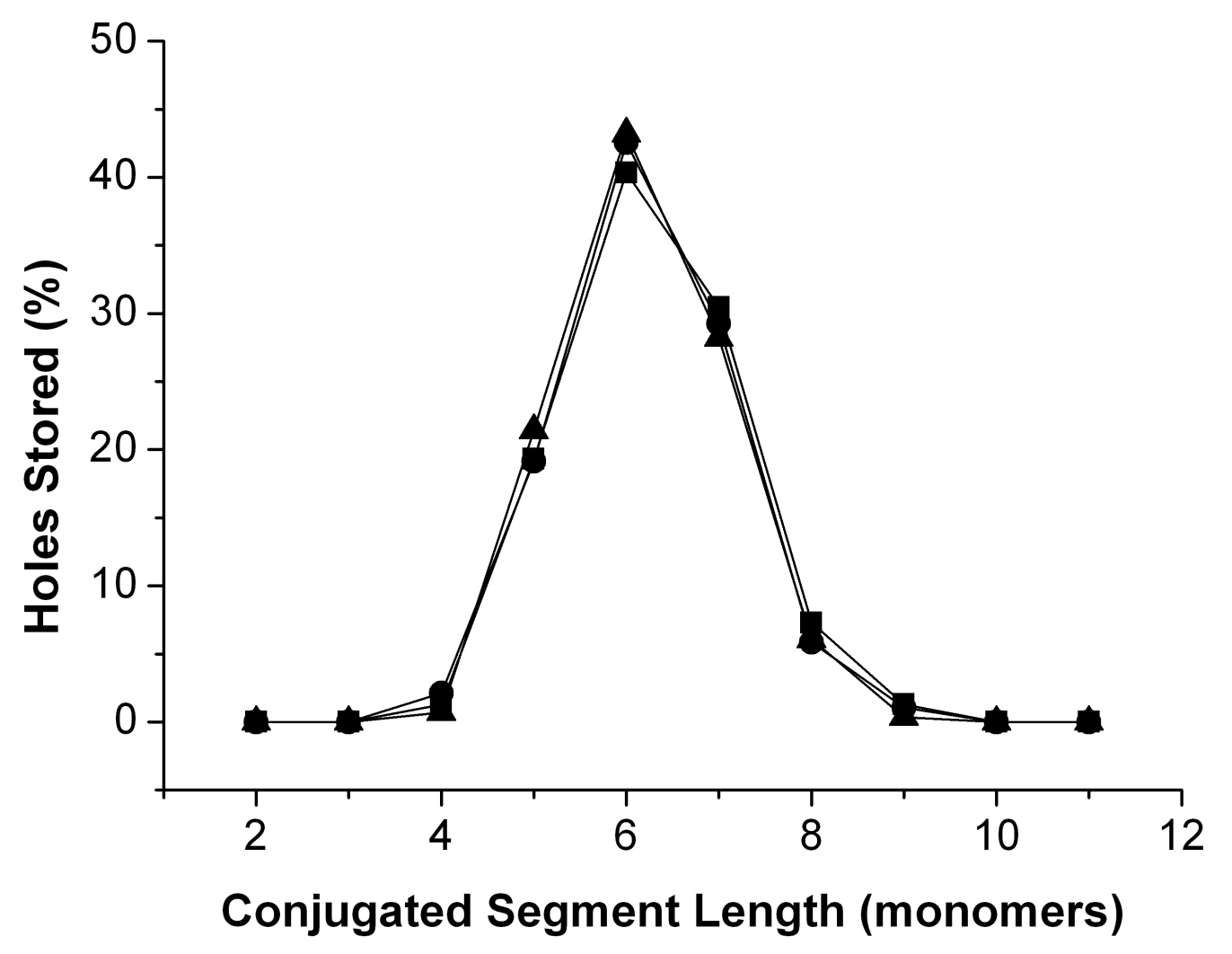


Figure 3

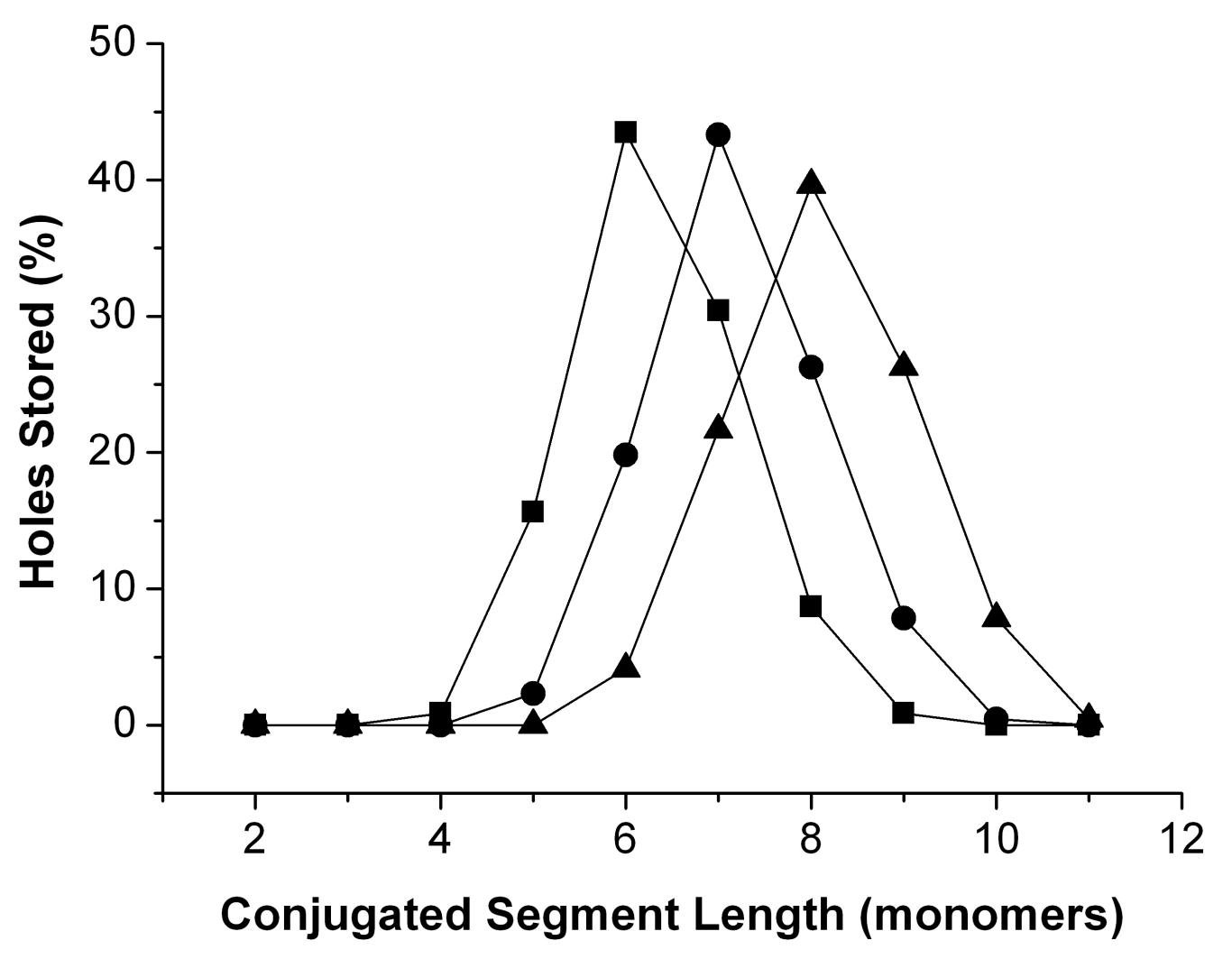


Figure 4

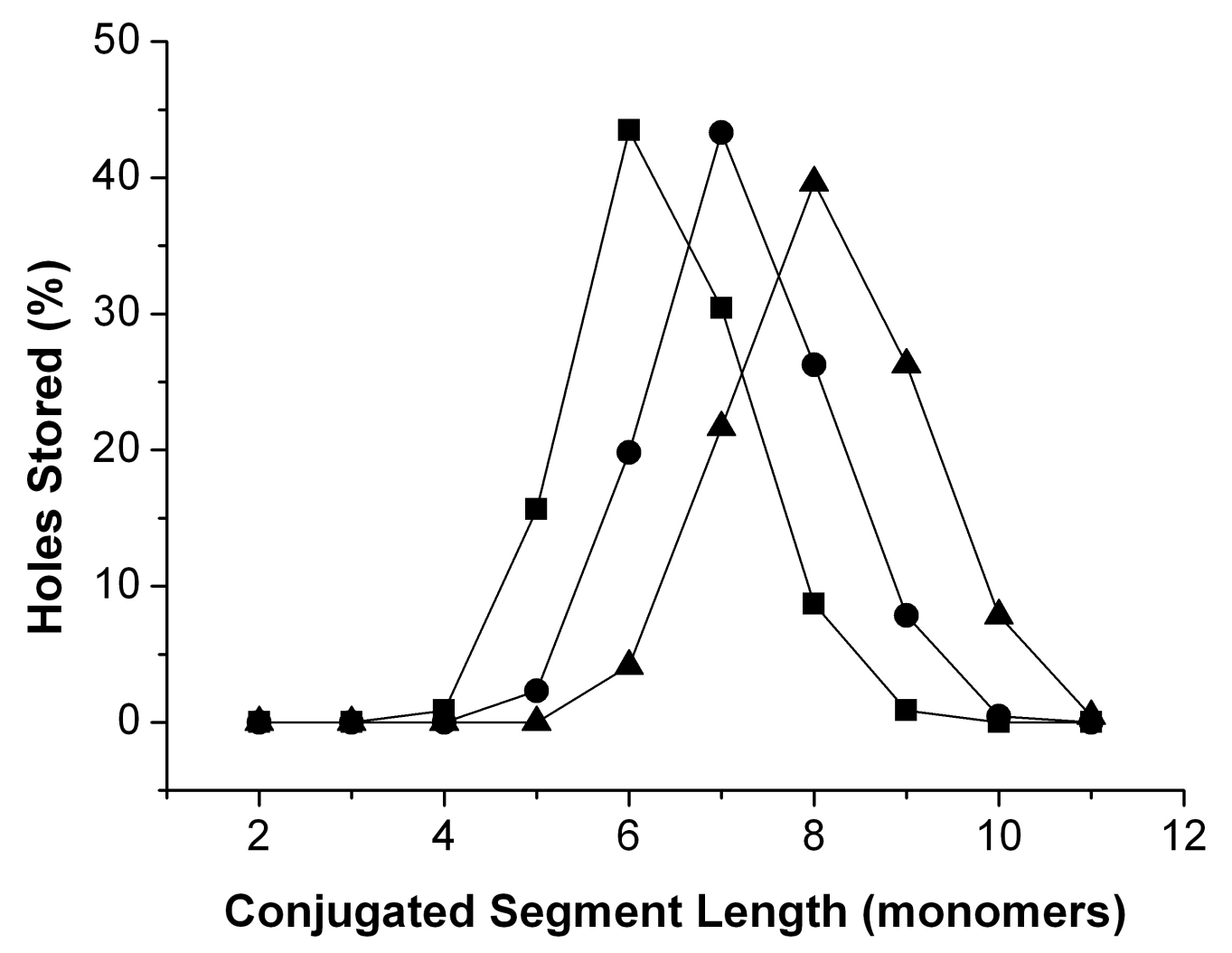

